\title{
Nonlinear Saffman-Taylor Instability
}

\author{
E. Álvarez-Lacalle, J. Ortín, and J. Casademunt \\ Departament ECM, Universitat de Barcelona, Avinguda Diagonal 647, E-08028, Barcelona, Spain
}

(Received 13 March 2003; published 2 February 2004)

\begin{abstract}
We show, both theoretically and experimentally, that the interface between two viscous fluids in a Hele-Shaw cell can be nonlinearly unstable before the Saffman-Taylor linear instability point is reached. We identify the family of exact elastica solutions [Nye et al., Eur. J. Phys. 5, 73 (1984)] as the unstable branch of the corresponding subcritical bifurcation which ends up at a topological singularity defined by interface pinchoff. We devise an experimental procedure to prepare arbitrary initial conditions in a Hele-Shaw cell. This is used to test the proposed bifurcation scenario and quantitatively asses its practical relevance.
\end{abstract}

DOI: 10.1103/PhysRevLett.92.054501

PACS numbers: 47.20.Ma, 47.20.Ky, 47.54.+r

The Saffman-Taylor (ST) instability [1,2], i.e., the fingering instability at the interface between two fluids of different viscosity confined within two closely spaced parallel plates, has received much attention in the last decades as a paradigm of interfacial pattern formation in nonequilibrium extended systems.

In this Letter we show that, before the Saffman-Taylor linear instability point is reached, the interface is already nonlinearly unstable, in the sense that finite-amplitude perturbations grow while infinitesimal ones decay. The corresponding unstable branch in this bifurcation scenario is defined by a family of undulating elastica solutions, the class of exact interfacial solutions first reported by Nye, Lean, and Wright [3] whose relevance becomes now apparent. This class of solutions and its associated bifurcation scenario is arguably valid also for other morphological instabilities (e.g., MullinsSekerka). In all cases, the subcritical unstable branch ends up at a topological singularity defined by interface pinchoff. Furthermore, the identification of the exact unstable branch allows a direct, quantitative experimental test of the observable dynamic implications of the above results. To this purpose, we have devised an original procedure that allows the preparation of arbitrary initial conditions in a Hele-Shaw (HS) cell à la carte.

We consider a rectangular HS cell, made of two parallel plates of width $L$, separated by a small gap $b \ll L$ (Fig. 1). In the generic case a less viscous fluid 1 (air) displaces a more viscous fluid 2 (oil), as the latter is withdrawn from one side at velocity $V_{\infty}$. We call $\mu_{1}, \mu_{2}, \rho_{1}, \rho_{2}$ the dynamic viscosities and densities of the two fluids, and $\sigma$ their interfacial tension.

A linear stability analysis of the planar interface [4] shows that the growth rate of a sinusoidal mode of wavelength $\lambda$ is $\omega(k)=C|k|\left(1-B k^{2}\right)$ where $k=2 \pi / \lambda$, and $B, C$ are given by $B=D / C, C=\left(b^{2} / 12\right)\left(\rho_{2}-\rho_{1}\right) g /$ $\left(\mu_{2}+\mu_{1}\right)+A V_{\infty}$, with $D=\left(b^{2} / 12\right) \sigma /\left(\mu_{2}+\mu_{1}\right), A=$ $\left(\mu_{2}-\mu_{1}\right) /\left(\mu_{2}+\mu_{1}\right)$, and we include the possibility of a gravity component $g$ in the $\hat{y}$ direction. The dispersion relation above shows that, for an infinite system, we are dealing with a long-wavelength instability, with the unstable band extending from $k=\sqrt{1 / B}$ to $k=0$.

At the nonlinear level, we show here that any given mode of wavelength $\lambda$ undergoes a subcritical bifurcation as the dimensionless parameter $B^{*}=B(2 \pi / \lambda)^{2}$ crosses unity, implying that finite-amplitude excitations of that mode may grow if they surpass a certain amplitude threshold, which depends on the distance to the bifurcation. This fact is relevant, for instance, in a finite system, resulting in a fully nonlinear instability if all modes allowed lie in the linearly stable band [5].

This scenario is captured by a weakly nonlinear analysis of a nearly marginal mode. Indeed, following Ref. [6], we obtain the amplitude equation

$$
\dot{\delta}=\left(1-B^{*}\right) \delta+\frac{3}{2}|\delta|^{2} \delta,
$$

where the dimensionless amplitude $\delta$ is measured in units of $2 \pi / \lambda$, and for simplicity we have used $C=1$ and $\lambda=$ $2 \pi$. The positive sign of the nonlinear term makes evident the subcritical nature of the bifurcation. The condition $\dot{\delta}=0$ defines a parabolic branch of unstable stationary solutions (Fig. 2). Remarkably, unlike most common situations, higher orders in the above equation will never saturate the growth to a finite amplitude, since for this problem (as for free solidification) the growth evolves towards steadily propagating configurations. It remains thus an open question how the above unstable branch is continued into the strongly nonlinear domain.

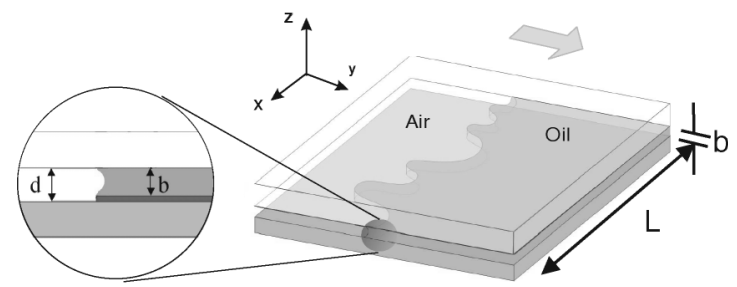

FIG. 1. Sketch of a rectangular Hele-Shaw cell of width $L$ and gap thickness $b$. The gap step used to prepare arbitrary initial conditions (see text) is also sketched. 


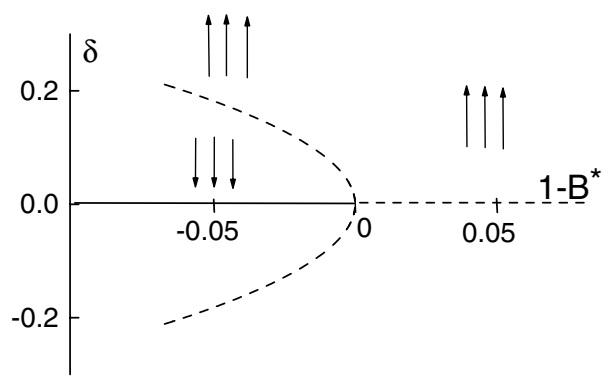

FIG. 2. Bifurcation diagram showing the stationary solutions of Eq. (1). The horizontal axis corresponds to the planar interface and the parabola to the subcritical branch. Linearly stable (unstable) solutions are represented by solid (dashed) lines.

Notice that this analysis predicts the existence of nontrivial steady solutions for finite surface tension. The only known solutions for the usual Saffman-Taylor problem with finite surface tension that we are aware of are the elastica solutions reported by Nye, Lean, and Wright [3]. They were found on the basis of simple arguments balancing capillary and hydrostatic pressure, as in pendant drops. Such solutions do correspond also to steady shapes propagating with velocity $V_{\infty}$ in the lab frame. They were used within an asymptotic matching scheme for the selection of the ST finger in Refs. [7,8]. We will show that these solutions are the exact continuation of the unstable branch discussed above. Solutions of this type are given in terms of nonlinear ordinary differential equations (ODE)s. For the channel geometry, for instance, one gets a uniparametric family of shapes which solve the pendulum equation

$$
B \theta_{s s}+\sin \theta=0,
$$

where $\theta$ is the angle between the normal direction and the $y$ axis, and $s$ is the arclength. A gallery of the resulting interfaces is displayed on the left panel of Fig. 3. Also shown is the maximum-to-minimum height of the interfaces, $H$, which parametrizes this family of solutions.

It may be surprising that solutions of a fully nonlocal dynamics may be represented by such a simple local equation, even for a nonstatic case. This apparent paradox is easily solved by realizing that the above type of solutions correspond to imposing the condition of zero vorticity in the vortex-sheet formalism of Trygvasson and Aref [9]. In the channel geometry and in a comoving frame with velocity $V_{\infty}$, the evolution of the interface position $\vec{r}(s, t)$, as a function of the interface arclength $s$ and time $t$, is given by $\dot{\vec{r}}(s, t) \cdot \hat{n}=\vec{U} \cdot \hat{n}$, where

$$
\vec{U}(s, t)=\frac{1}{2 \pi} \mathrm{P} \int \frac{\hat{z} \times\left[\vec{r}(s, t)-\vec{r}\left(s^{\prime}, t\right)\right]}{\left|\vec{r}(s, t)-\vec{r}\left(s^{\prime}, t\right)\right|^{2}} \gamma\left(s^{\prime}, t\right) d s^{\prime},
$$

where $\mathrm{P}$ denotes the principal value of the integral, $\gamma=$ $2 A(\vec{U} \cdot \hat{\mathbf{s}})+2 C \hat{\mathbf{y}} \cdot \hat{\mathbf{s}}+2 D \kappa_{s}$ is the vorticity, and $\kappa_{s}$ the derivative of the in-plane local curvature of the interface.

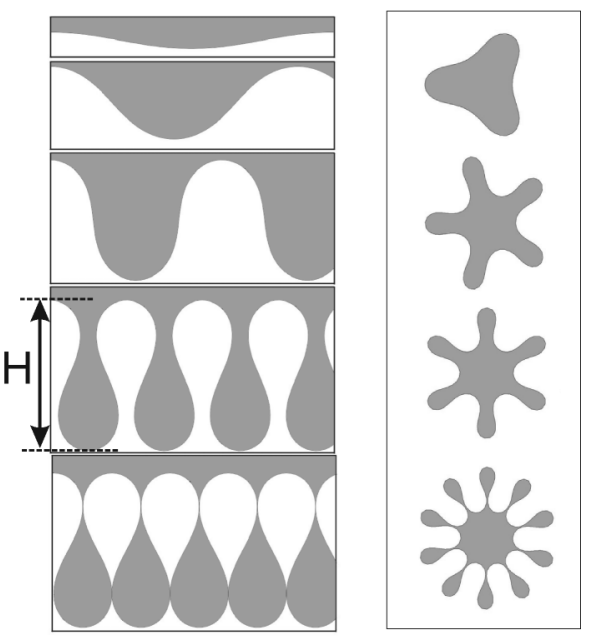

FIG. 3. Exact stationary solutions of the ST problem with nonzero surface tension, in channel geometry (left) and circular geometry with rotation (right).

The condition $\gamma=0=2 C \hat{\mathbf{y}} \cdot \hat{\mathbf{s}}+2 D \kappa_{s}($ with $\vec{U}=0)$ is precisely Eq. (2). This automatically solves the equation for the interface deformation, $\dot{\vec{r}}=0$, while it does not rule out a constant velocity field.

The condition of zero vorticity yields a simple way to generalize the solutions to other cases. One relevant example is a radial HS cell with rotation [10,11]. The ODE that defines the condition $\gamma=0$ in this case balances capillary and centrifugal forces, and reads

$$
\kappa_{\phi}-\frac{\left(\rho_{2}-\rho_{1}\right) \Omega^{2}}{\sigma} r r_{\phi}=0
$$

in polar coordinates, where $\Omega$ is the angular velocity of the cell and the inner fluid 2 has been supposed more dense than the outer fluid 1. A sample of corresponding interfaces is displayed on the right panel of Fig. 3. Notice, however, that when fluid injection is present viscous pressure and capillary pressure can be balanced only for a given time, and thus not yield steady shapes.

The elastica solutions given in Eq. (2) are independent of $A$, and surface tension, though necessary, only sets the spatial scale. For a limiting value of the parameter of the family, the solution presents a topological singularity, implying an interface pinchoff (left bottom plot in Fig. 3). Beyond that the curves obtained intersect and thus have no longer physical meaning for the problem at hand.

A direct expansion of the solutions of Eq. (2) in the amplitude $\delta$ shows that they coincide to lowest order with those of Eq. (1) (see Fig. 4), implying that the elastica solutions are the continuation of the subcritical branch originating at the bifurcation. That is, each point of the subcritical branch is one interface of the family of elastica solutions for a given $\lambda$. Notice that the continuation of the exact curve into the nonlinear domain in Fig. 4 is 


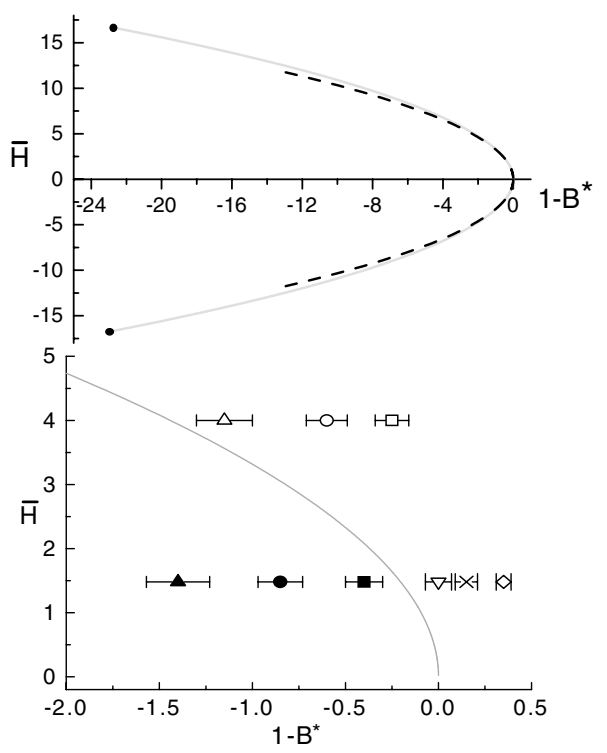

FIG. 4. In the top panel, the distance $H$ between the maximum and the minimum height of the interface is plotted vs $B$, in scaled variables $\bar{H}=H(2 \pi / \lambda), B^{*}=B(2 \pi / \lambda)^{2}$, up to $B^{*}=3$. The branch of elastica solutions (subcritical) is represented by a light solid line. The branch ends at $B^{*}=23.9$, where interface pinchoff occurs. The dashed line is the weakly nonlinear approximation obtained from Eq. (1). The bottom panel shows a magnification of the upper unstable branch, together with the set of initial conditions used in our experiments, represented by different symbols.

parametrized only with $H$. Obviously this is an incomplete description since, beyond the center manifold reduction, the problem is infinite-dimensional. The curve shown is thus only a subset of the set of interface configurations that separates the basins of attraction of the two relevant attractors, namely, the planar interface and the Saffman-Taylor finger. Nevertheless, perturbations of the elastica solutions leading to interface pinchoff cannot be ruled out, so the actual structure of such separatrix of basins of attraction is presumably complex and remains unknown. In any case, we expect that the fact that the continuation of the subcritical unstable branch ends up at a topological singularity is a generic feature. For instance, the dynamics of free (isotropic) solidification with small Péclet number is known to reduce to $A=1$ viscous fingering [8]. It is thus reasonable to expect, on topological grounds, that the elastica solutions will also approximate the corresponding subcritical branch of the solidification problem, and that the qualitative scenario will be the same.

In order to test quantitatively the dynamic scenario described above, and to demonstrate its relevance in practice, we have carried out a series of experiments in a horizontal HS channel. These experiments are also intended to probe the robustness of a description of the phase space based solely on the height of the curve as a finite-amplitude criterion for the nonlinear instability.
The cell is made of two glass plates, of $15 \mathrm{~mm}$ thickness and $250 \mathrm{~mm}$ lateral size, separated by a brass frame of thickness $b=0.50 \mathrm{~mm}$. It is originally filled with a silicone oil Rhodorsil 47V10 (dynamic viscosity $\mu=$ $8.4 \times 10^{-3} \mathrm{~Pa} \cdot \mathrm{s}$, density $\rho=930 \mathrm{~kg} / \mathrm{m}^{3}$, and oil-air surface tension $\sigma=20.7 \times 10^{-3} \mathrm{~N} / \mathrm{m}$, at $20^{\circ} \mathrm{C}$ ). In the experiment the oil is withdrawn at a constant volumetric rate from one side of the cell, using a syringe pump, allowing air to enter the cell from the other side.

Essential to these experiments is the ability to generate initial conditions of prescribed shapes. To this aim we have developed an original procedure that exploits the sensitivity of capillary pressure to slight gap thickness variations. The gap of the Hele-Shaw cell on the region initially occupied by the oil (displaced fluid) is made slightly smaller (thickness $b$ ) than on the region occupied by air (displacing fluid) (thickness $d$ ), and the step between the two regions follows the shape of the desired initial condition (Fig. 1). Once the oil has been gently introduced in the cell, the small difference in capillary pressure across the interface suffices to hold the interface still, following the profile of the gap step, until oil withdrawal starts. In our experiment the gap thickness is modified by attaching a thin film of polyethylene (Alkor-Decor, $d-b=0.1 \mathrm{~mm}$ thickness) to the region of the bottom plate to be covered initially by the oil. One end of the plastic film is cut carefully with the shape of the desired initial condition.

Figure 5 shows a series of photographs taken in two different experiments. In both cases the initial condition, shown as a dashed line, has been designed as follows. The central part, of lateral size $L / 3$, corresponds to an elastica curve with $\lambda=L / 3$. This is continued on each side with a

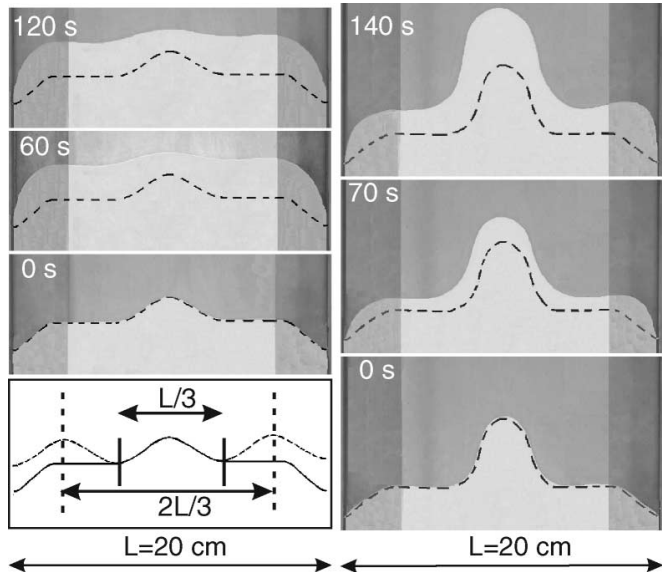

FIG. 5. Snapshots of two experiments for two different initial conditions. Dark and light gray regions, above and below the interface, correspond, respectively, to silicone oil and air. A dashed line represents the initial condition, designed as sketched on the bottom left panel (see text for details). The shaded strips on the sides define the regions disregarded in the quantitative analysis. 


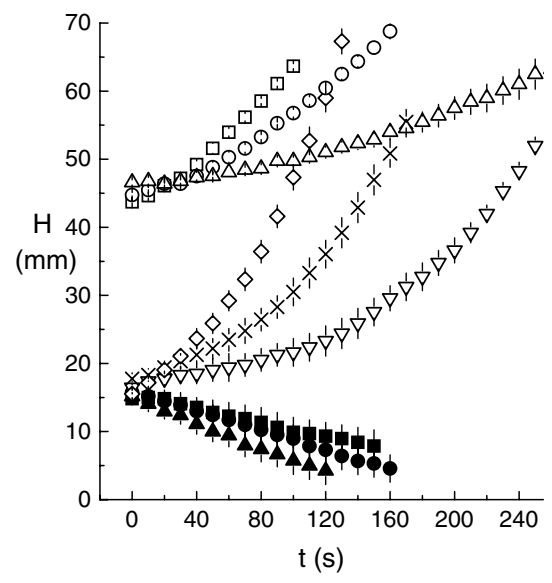

FIG. 6. Time evolution of the measured maximum-tominimum interface height for different experiments. The experimental parameters are indicated in Fig. 4 with corresponding symbols.

flat section of size $L / 6$, and terminated with a receding section, also of size $L / 6$ that meets the side walls perpendicularly. This particular form of the initial condition is designed to minimize the influence of the boundaries, which may be critical in these experiments [12]. The elastica curve taken as initial condition in the first experiment (left) has $\bar{H}=1.5$, while the second one (right) has $\bar{H}=4$.0. Both experiments have been carried out at the same injection rate $Q=65 \mathrm{ml} / \mathrm{h}$, which corresponds to a nominal $B^{*}=1.7$ [13]. Thus the conditions in the first (second) experiment fall below (above) the branch of Fig. 4 , as shown in the bottom panel of Fig. 4 as solid (open) circles. If the separatrix of the different basins of attraction in phase space, for initial conditions close to elastica interfaces, is sufficiently well described by the branch of elastica solutions, experiments located initially below this branch should evolve towards the flat interface, and experiments located initially above should grow towards the ST finger [14]. This is exactly what is observed in the experiments shown in Fig. 5. On the left, the interface has become nearly flat after $120 \mathrm{~s}$, as predicted. On the right, the maximum-to-minimum interface height keeps growing with time.

We have repeated the same kind of experiments using the two initial conditions of Fig. 5 and different injection rates (i.e., different $B^{*}$ ). The actual values of $\bar{H}$ (in the initial condition) and $B^{*}$, for the nine experiments performed, are shown in Fig. 4. The growth or decay of the maximum-to-minimum height of the interface with time in these experiments is shown in Fig. 6. Our experimental results show conclusively that all the interfaces decay or grow depending on whether they are, respectively, below or above the branch of solutions depicted in Fig. 4.

In conclusion: (i) Our experiments provide robust evidence that linearly stable modes in the ST problem are generically nonlinearly unstable. Consequently, in spe- cific situations the ST instability is globally nonlinear. (ii) We have identified the elastica solutions of the ST problem with the exact unstable branch of the subcritical bifurcation diagram. (iii) Experiments also show that this branch is an appropriate projection of the separatrix between the basins of attraction of the ST finger and the planar interface, even far from the bifurcation point. (iv) This branch ends at a topological singularity (interface pinchoff).

The close connection between the ST problem and related interfacial growth problems, together with the generic nature of our bifurcation analysis, suggest that the qualitative features of the above conclusions are relevant to a variety of problems.

We are grateful to one referee for pointing out that the elastica solutions were first reported in Ref. [3]. This work has been supported by projects BXX2000-0638-C02-02 (MCyT, Spain), SGR-2000-00433 (DURSI, Generalitat de Catalunya), and HPRN-CT-2002-00312 (European Commission). E. A. L. and J. O. acknowledge the DURSI for additional financial support.

*Electronic address: enric@ecm.ub.es

[1] P. G. Saffman and G. I. Taylor, Proc. R. Soc. London Ser. A 245, 312 (1958).

[2] D. Bensimon et al., Rev. Mod. Phys. 58, 977 (1986).

[3] J. F. Nye, H.W. Lean, and A. N. Wright, Eur. J. Phys. 5, 73 (1984).

[4] R. L. Chuoke, P. van Meurs, and C. van der Poel, Trans. Metall. Soc. AIME 216, 188 (1959).

[5] A different nonlinear instability was already reported for the ST problem modified by the addition of surfactants by H. Guo, D. C. Hong, and D. A. Kurtze, Phys. Rev. Lett. 69, 1520 (1992); Phys. Rev. E 51, 4469 (1995).

[6] E. Álvarez-Lacalle, J. Casademunt, and J. Ortín, Phys. Rev. E 64, 016302 (2001); (to be published).

[7] Y. Pomeau, Stud. Appl. Math. 73, 75 (1985).

[8] T. Dombre and V. Hakim, Phys. Rev. A 36, 2811 (1987).

[9] G. Trygvasson and H. Aref, J. Fluid Mech. 136, 1 (1983).

[10] L.W. Schwartz, Phys. Fluids A 1, 167 (1989).

[11] L. Carrillo et al., Phys. Rev. E 54, 6260 (1996).

[12] The front position tends to pin at the side walls, giving rise to the systematic formation of one air finger at each side. By using this particular form of the initial condition, we are able to prevent the formation of lateral fingers during the time interval relevant for the experiments.

[13] Wall effects slightly modify the average velocity of the central part, affecting the actual value of $B^{*}$. Small changes in the initial value of $\lambda$ also have an influence on $B^{*}$. Both facts are taken into account to compute the actual values of $B^{*}$, reported in Fig. 4. The error bars account for the experimental uncertainties in $b$ and $\mu$.

[14] To what extent the projection of the separatrix into the elastica branch is appropriate for initial interfaces far from the elastica shapes is an open question. 